\title{
Real Hypersurfaces in the Complex Projective Plane Satisfying an Equality Involving $\delta(2)$
}

\author{
Toru Sasahara \\ (Communicated by Bang-Yen Chen)
}

\begin{abstract}
It was proved in Chen's paper (Arch. Math. (Basel) 67 (1996), 519-528) that every real hypersurface in the complex projective plane of constant holomorphic sectional curvature 4 satisfies

$$
\delta(2) \leq \frac{9}{4} H^{2}+5
$$

where $H$ is the mean curvature and $\delta(2)$ is a $\delta$-invariant introduced by him. In this paper, we study non-Hopf real hypersurfaces satisfying the equality case of the inequality under the condition that the mean curvature is constant along each integral curve of the Reeb vector field. We describe how to obtain all such hypersurfaces.
\end{abstract}

\section{Introduction}

For a Riemannian $m$-manifold $M$ with $m>2$, Chen [2] introduced in the early 1990s the following invariant:

$$
\delta(2)(p)=\tau(p)-\inf \left\{K(\pi) \mid \pi \text { is a plane in } T_{p} M\right\},
$$

where $\tau$ is the scalar curvature and $K(\pi)$ is the sectional curvature of $\pi$. If $m=3$, then $\delta(2)(p)$ is equal to the maximum Ricci curvature function $\overline{\operatorname{Ric}}$ on $M$ defined by $\overline{\operatorname{Ric}}(p)=\max \left\{S(X, X) \mid X \in T_{p} M,\|X\|=1\right\}$, where $S$ is the Ricci tensor. For general $\delta$-invariants, see [4] for details.

It was proved in [3] that every real hypersurface in the complex projective space $\mathbb{C} P^{n}$ of complex dimension $n$ and constant holomorphic sectional curvature 4 satisfies

$$
\delta(2) \leq \frac{(2 n-1)^{2}(2 n-3)}{4(n-1)} H^{2}+2 n^{2}-3,
$$

where $H$ denotes the mean curvature. A real hypersurface in $\mathbb{C} P^{n}$ is said to be $\delta(2)$-ideal if it attains equality in (1.1) at each point. Chen [3] completely classified $\delta(2)$-ideal Hopf real hypersurfaces in $\mathbb{C} P^{n}$. In [7], the author proved that a non-Hopf real hypersurface with constant mean curvature in $\mathbb{C} P^{2}$ is $\delta(2)$-ideal if and only if it is a minimal ruled real hypersurface. In this paper, we classify $\delta(2)$-ideal non-Hopf real hypersurfaces in $\mathbb{C} P^{2}$ whose mean curvature is constant along each integral curve of the Reeb vector field. 


\section{Preliminaries}

Let $M$ be a real hypersurface in the complex projective space $\mathbb{C} P^{n}$. We denote by $J$ the almost complex structure of $\mathbb{C} P^{n}$. For a unit normal vector field $N$, the vector field on $M$ defined by $\xi=-J N$ is called the Reeb vector field. If $\xi$ is a principal curvature vector at every point of $M$, then $M$ is said to be Hopf.

Let $\mathcal{H}$ be the holomorphic distribution defined by $\mathcal{H}=\bigcup_{p \in M}\left\{X \in T_{p} M \mid\langle X, \xi\rangle=0\right\}$, where $\langle\cdot, \cdot\rangle$ denotes the metric of $\mathbb{C} P^{n}$. If $\mathcal{H}$ is integrable and each leaf of its maximal integral manifolds is a totally geodesic complex hypersurface, then $M$ is said to be ruled.

Denote by $\nabla$ and $\tilde{\nabla}$ the Levi-Civita connections on $M$ and $\mathbb{C} P^{n}$, respectively. The Gauss and Weingarten formulas are respectively given by

$$
\begin{aligned}
\tilde{\nabla}_{X} Y & =\nabla_{X} Y+\langle A X, Y\rangle N, \\
\tilde{\nabla}_{X} N & =-A X
\end{aligned}
$$

for tangent vector fields $X, Y$ and a unit normal vector field $N$, where $A$ is the shape operator with respect to $N$. The function $H=\operatorname{tr} A /(2 n-1)$ is called the mean curvature. If it vanishes identically, then $M$ is said to be minimal.

For any vector field $X$ tangent to $M$, we denote the tangential component of $J X$ by $\phi X$. Then by the Gauss and Weingarten formulas, we have

$$
\nabla_{X} \xi=\phi A X
$$

We denote by $R$ the Riemannian curvature tensor of $M$. Then, the equations of Gauss and Codazzi are respectively given by

$$
\begin{array}{r}
R(X, Y) Z=\langle Y, Z\rangle X-\langle X, Z\rangle Y+\langle\phi Y, Z\rangle \phi X-\langle\phi X, Z\rangle \phi Y \\
-2\langle\phi X, Y\rangle \phi Z+\langle A Y, Z\rangle A X-\langle A X, Z\rangle A Y \\
\left(\nabla_{X} A\right) Y-\left(\nabla_{Y} A\right) X=\langle X, \xi\rangle \phi Y-\langle Y, \xi\rangle \phi X-2\langle\phi X, Y\rangle \xi
\end{array}
$$

\section{3. $\delta(2)$-ideal real hypersurfaces}

Applying [3, Theorem 2] to real hypersurfaces in $\mathbb{C} P^{n}$, we have the following general inequality.

Theorem 3.1. Let $M$ be a real hypersurface in $\mathbb{C} P^{n}$. For any point $p \in M$ and any plane $\pi \subset T_{p} M$, we have

$$
\tau-K(\pi) \leq \frac{(2 n-1)^{2}(2 n-3)}{4(n-1)} H^{2}+2 n^{2}-3-3\left\langle J e_{1}, e_{2}\right\rangle^{2},
$$

where $\left\{e_{1}, e_{2}\right\}$ is an orthonormal basis of $\pi$. The equality sign in (3.1) holds at a point $p \in M$ if and only if there exists an orthonormal basis $\left\{e_{1}, e_{2}, \ldots, e_{2 n-1}\right\}$ at $p$ such that the shape operator at $p$ is represented by a matrix

$$
A=\left(\begin{array}{ccccc}
\alpha & \beta & 0 & \ldots & 0 \\
\beta & \gamma & 0 & \ldots & 0 \\
0 & 0 & \mu & \ldots & 0 \\
\vdots & \vdots & \vdots & \ddots & \vdots \\
0 & 0 & 0 & \ldots & \mu
\end{array}\right)
$$

where $\alpha+\gamma=\mu$.

We note that $\left\langle J e_{1}, e_{2}\right\rangle^{2}$ in (3.1) is independent of the choice of the orthonormal basis $\left\{e_{1}, e_{2}\right\}$ of $\pi$.

The following Corollary immediately follows from Theorem 3.1.

Corollary 3.1 ([3]). Let $M$ be a real hypersurface in $\mathbb{C} P^{n}$. Then, we have

$$
\delta(2) \leq \frac{(2 n-1)^{2}(2 n-3)}{4(n-1)} H^{2}+2 n^{2}-3
$$

at each point of $M$. The equality sign in (3.3) holds at a point $p \in M$ if and only if there exists an orthonormal basis $\left\{e_{1}, e_{2}, \ldots, e_{2 n-1}\right\}$ at $p$ such that

(1) $\left\langle J e_{1}, e_{2}\right\rangle=0$,

(2) $K\left(e_{1} \wedge e_{2}\right)=\inf K$,

(3) the shape operator at $p$ is represented by a matrix (3.2) with $\alpha+\gamma=\mu$. 
Remark 3.1. It follows from (3.1) that if (1) and (3) in Corollary 3.1 hold, then (2) is automatically satisfied.

A real hypersurface in $\mathbb{C} P^{n}$ is said to be $\delta(2)$-ideal if it attains equality in (3.3) at each point. In [3], Chen proved that a Hopf real hypersurface in $\mathbb{C} P^{n}$ is $\delta(2)$-ideal if and only if it is an open part of one of the following hypersurfaces: (i) a geodesic sphere with radius $\pi / 4$ in $\mathbb{C} P^{n}$, (ii) a tubular hypersurface with radius $r=\tan ^{-1}((1+\sqrt{5}-\sqrt{2+2 \sqrt{5}}) / 2)$ over a complex quadric curve $Q_{1}$ in $\mathbb{C} P^{2}$.

We now present a class of $\delta(2)$-ideal non-Hopf hypersurfaces in $\mathbb{C} P^{2}$.

Example 3.1. Suppose that $\alpha(s), \beta(s), \gamma(s)$ and $\mu(s)$ satisfy

$$
\begin{aligned}
& \alpha^{\prime}=\beta(\alpha+\gamma-3 \mu), \\
& \beta^{\prime}=\beta^{2}+\gamma^{2}+\mu(\alpha-2 \gamma)+1, \\
& \gamma^{\prime}=\frac{(\gamma-\mu)\left(\gamma^{2}-\alpha \gamma-1\right)}{\beta}+\beta(2 \gamma+\mu),
\end{aligned}
$$

on an open interval $I \subset \mathbb{R}$, where $\beta(s)$ are nowhere zero. According to Theorem 5 in [5], there exists a smooth immersion $\Phi: I \times \mathbb{R}^{2} \rightarrow \mathbb{C} P^{2}$ determining a non-Hopf real hypersurface in $\mathbb{C} P^{2}$, such that the shape operator $A$ is represented by (3.2) with respect to an orthonormal frame field $\{\xi, X, \phi X\}$, where $\phi X=\partial / \partial s$. The distribution $\mathcal{D}$ spanned by $\xi$ and $X$ is integrable, and $\Phi$ maps the $\mathbb{R}^{2}$-factors onto the $\mathcal{D}$-leaves. Clearly, the mean curvature of the hypersurface is constant along each integral curve of the Reeb vector field.

If $\alpha+\gamma=\mu$ on $I$, then Corollary 3.1 and Remark 3.1 imply that $\Phi$ is $\delta(2)$-ideal. In particular, if $\alpha=\gamma=\mu=0$ on $I$, then $\operatorname{tr} A=0$ and $\langle A X, Y\rangle=0$ for any tangent vector field $X, Y$ on $M$ orthogonal to $\xi$, and hence $\Phi$ is minimal ruled (see [1, p.445] and [6]).

Remark 3.2. Substitution of $\alpha+\gamma=\mu$ into (3.4) gives a autonomous system. It follows from Picard's theorem that for given initial values $\alpha\left(s_{0}\right)=\alpha_{0}, \beta\left(s_{0}\right)=\beta_{0}, \gamma\left(s_{0}\right)=\gamma_{0}$ with $\beta_{0} \neq 0$ and $\alpha_{0}+\gamma_{0} \neq 0$, the initial value problem of (3.4) with $\alpha+\gamma=\mu$ has a unique solution satisfying $\beta \neq 0$ and $\alpha+\gamma \neq 0$ on some open interval containing $s_{0}$. Therefore, there exist infinity many $\delta(2)$-ideal real hypersurfaces in $\mathbb{C} P^{2}$ which are non-Hopf and non-minimal.

Remark 3.3. Let $M$ be a real hypersurface in the complex hyperbolic space $\mathbb{C} H^{n}$ of constant holomorphic sectional curvature -4 . Then we have

$$
\delta(2) \leq \frac{(2 n-1)^{2}(2 n-3)}{4(n-1)} H^{2}+6-2 n^{2} .
$$

The equality sign of the inequality holds identically if and only if $M$ is an open part of the horosphere in $\mathbb{C} H^{2}$ (see [3]).

\section{Main result}

The following theorem is the main result of this paper.

Theorem 4.1. Let $M$ be a $\delta(2)$-ideal non-Hopf real hypersurface in $\mathbb{C} P^{2}$. If the mean curvature is constant along each integral curve of the Reeb vector field, then $M$ is locally obtained by the construction described in Example 3.1.

Proof. Let $M$ be a $\delta(2)$-ideal non-Hopf real hypersurface in $\mathbb{C} P^{2}$. Let $\left\{e_{1}, e_{2}, e_{3}\right\}$ be a local orthonormal frame field described in Corollary 3.1. We put $\xi=p e_{1}+q e_{2}+r e_{3}$ for some functions $p, q$ and $r$. It follows from $\left\langle J e_{1}, e_{2}\right\rangle=0$ that $r\left\langle J e_{3}, e_{1}\right\rangle=r\left\langle J e_{3}, e_{2}\right\rangle=0$. If $r \neq 0$, then $\xi=e_{3}$. However, this contradicts $\left\langle J e_{1}, e_{2}\right\rangle=0$. Hence, $r=0$ holds, that is, $\xi$ lies in $\operatorname{Span}\left\{e_{1}, e_{2}\right\}$. We may assume that $e_{1}=\xi$ and $J e_{2}=e_{3}$. From (3) of Corollary 3.1, we see that the shape operator satisfies the following:

$$
A \xi=(\mu-\gamma) \xi+\beta e_{2}, A e_{2}=\gamma e_{2}+\beta \xi, A e_{3}=\mu e_{3} .
$$

Let $\Omega$ be an open set where $\beta \neq 0$. We work in $\Omega$. Using (2.1) and (4.1), we get

$$
\nabla_{e_{2}} \xi=\gamma e_{3}, \quad \nabla_{e_{3}} \xi=-\mu e_{2}, \quad \nabla_{\xi} \xi=\beta e_{3} .
$$

Since $\left\langle\nabla e_{i}, e_{j}\right\rangle=-\left\langle\nabla e_{j}, e_{i}\right\rangle$ holds, by (4.2) we have

$$
\begin{aligned}
& \nabla_{e_{2}} e_{2}=\kappa_{1} e_{3}, \quad \nabla_{e_{3}} e_{2}=\kappa_{2} e_{3}+\mu \xi, \quad \nabla_{\xi} e_{2}=\kappa_{3} e_{3}, \\
& \nabla_{e_{2}} e_{3}=-\kappa_{1} e_{2}-\gamma \xi, \quad \nabla_{e_{3}} e_{3}=-\kappa_{2} e_{2}, \quad \nabla_{\xi} e_{3}=-\kappa_{3} e_{2}-\beta \xi
\end{aligned}
$$


for some functions $\kappa_{1}, \kappa_{2}$ and $\kappa_{3}$.

Assume that the mean curvature $H=\mu / 3$ is constant along each integral curve of the Reeb vector field $\xi$, that is,

$$
\xi \mu=0 \text {. }
$$

From (4.1), (4.2), (4.3) and the equation (2.3) of Codazzi, it follows that

$$
\begin{aligned}
e_{2} \mu & =0, \\
e_{3} \gamma & =(\gamma-\mu) \kappa_{1}+\beta(\gamma+2 \mu), \\
e_{3} \beta & =-\gamma^{2}+\beta \kappa_{1}-2 \gamma \mu+\mu^{2}+2, \\
e_{2} \beta & =\xi \gamma, \\
e_{2} \gamma & =-\xi \beta, \\
\beta \kappa_{1}+(\mu-\gamma) \kappa_{3} & =\beta^{2}+\gamma^{2}-1, \\
\kappa_{2} & =0, \\
e_{3}(\mu-\gamma) & =\beta\left(\kappa_{3}-2 \mu-\gamma\right) .
\end{aligned}
$$

Taking into account (4.11), the equation (2.2) of Gauss for $\left\langle R\left(e_{2}, e_{3}\right) e_{3}, e_{2}\right\rangle$ and $\left\langle R\left(\xi, e_{2}\right) e_{3}, e_{2}\right\rangle$ yields

$$
\begin{aligned}
e_{3} \kappa_{1} & =2 \mu \gamma+\kappa_{1}^{2}+(\gamma+\mu) \kappa_{3}+4, \\
\xi \kappa_{1} & =e_{2} \kappa_{3} .
\end{aligned}
$$

Using (4.2), (4.3), (4.4) and (4.5) we have

$$
0=\left[e_{2}, \xi\right] \mu=\left(\nabla_{e_{2}} \xi-\nabla_{\xi} e_{2}\right) \mu=\left(\gamma-\kappa_{3}\right) e_{3} \mu .
$$

Thus, we obtain that $\gamma=\kappa_{3}$ or $e_{3} \mu=0$.

Case (a): $e_{3} \mu=0$ on an open subset $U \subset \Omega$. In this case, combining (4.4) and (4.5) implies that $\mu$ is constant, that is, the mean curvature is constant on $U$. Hence, by virtue of [7, Theorem 1.2], we conclude that $U$ is minimal ruled.

Case (b): $\gamma=\kappa_{3}$ on an open subset $V \subset \Omega$. In this case, since $\nabla_{e_{2}} \xi-\nabla_{\xi} e_{2}=0$ holds, the distribution $\mathcal{D}$ spanned by $\xi$ and $e_{2}$ is integrable. Eliminating $e_{3} \gamma$ from (4.6) and (4.12), we obtain

$$
e_{3} \mu=(\gamma-\mu) \kappa_{1}+\beta \gamma
$$

Equations (4.10) and (4.13) become

$$
\begin{aligned}
\beta \kappa_{1} & =\beta^{2}+2 \gamma^{2}-\mu \gamma-1, \\
e_{3} \kappa_{1} & =\kappa_{1}^{2}+\gamma^{2}+3 \gamma \mu+4,
\end{aligned}
$$

respectively. From (4.9) and (4.14), it follows that

$$
\xi \kappa_{1}=-\xi \beta
$$

Elimination of $\kappa_{1}$ from (4.7) and (4.17) leads to

$$
e_{3} \beta=\beta^{2}+\gamma^{2}-3 \gamma \mu+\mu^{2}+1 .
$$

Using (4.2), (4.3), (4.6), (4.8), (4.11), (4.19) and (4.20), we have the following:

$$
\begin{aligned}
e_{3}(\xi \beta) & =\left(\nabla_{e_{3}} \xi-\nabla_{\xi} e_{3}\right) \beta+\xi\left(e_{3} \beta\right) \\
& =(\gamma-\mu) \xi \gamma+\beta(\xi \beta)+\xi\left(\beta^{2}+\gamma^{2}-3 \gamma \mu+\mu^{2}+1\right) \\
& =3 \beta(\xi \beta)+(3 \gamma-4 \mu) \xi \gamma \\
e_{3}(\xi \gamma) & =\left(\nabla_{e_{3}} \xi-\nabla_{\xi} e_{3}\right) \gamma+\xi\left(e_{3} \gamma\right) \\
& =(\mu-\gamma) \xi \beta+\beta(\xi \gamma)+\xi\left[(\gamma-\mu) \kappa_{1}+\beta(\gamma+2 \mu)\right] \\
& =(4 \mu-\gamma) \xi \beta+\left(2 \beta+\kappa_{1}\right) \xi \gamma .
\end{aligned}
$$


Differentiating (4.17) with respect to $\xi$, and using (4.4) and (4.19), we obtain

$$
\left(\kappa_{1}-3 \beta\right) \xi \beta+(\mu-4 \gamma) \xi \gamma=0 .
$$

Moreover, differentiating (4.23) with respect to $e_{3}$, we have

$$
\left(e_{3} \kappa_{1}-3 e_{3} \beta\right) \xi \beta+\left(\kappa_{1}-3 \beta\right) e_{3}(\xi \beta)+\left(e_{3} \mu-4 e_{3} \gamma\right) \xi \gamma+(\mu-4 \gamma) e_{3}(\xi \gamma)=0 .
$$

Substitution of (4.6), (4.16), (4.18), (4.20), (4.21) and (4.22) into (4.24) gives

$$
\left(\kappa_{1}^{2}-12 \beta^{2}+2 \gamma^{2}+\mu^{2}-5 \mu \gamma+3 \beta \kappa_{1}+1\right) \xi \beta+\left(6 \beta \mu-20 \beta \gamma-4 \gamma \kappa_{1}\right) \xi \gamma=0 .
$$

Equations (4.23) and (4.25) could be rewritten as

$$
\left(\begin{array}{ll}
a_{11} & a_{12} \\
a_{21} & a_{22}
\end{array}\right)\left(\begin{array}{l}
\xi \beta \\
\xi \gamma
\end{array}\right)=\left(\begin{array}{l}
0 \\
0
\end{array}\right)
$$

where the components of the square matrix are given by

$$
\begin{aligned}
& a_{11}=\kappa_{1}-3 \beta, \\
& a_{12}=\mu-4 \gamma, \\
& a_{21}=\kappa_{1}^{2}-12 \beta^{2}+2 \gamma^{2}+\mu^{2}-5 \mu \gamma+3 \beta \kappa_{1}+1, \\
& a_{22}=6 \beta \mu-20 \beta \gamma-4 \gamma \kappa_{1} .
\end{aligned}
$$

We divide Case (b) into two subcases.

Case (b.1): $a_{11} a_{22}-a_{21} a_{12} \neq 0$ on an open neighborhood $V_{1}$ of a point $p \in V$. In this case, by (4.26), we have $\xi \beta=\xi \gamma=0$. It follows from (4.8) and (4.9) that $e_{2} \beta=e_{2} \gamma=0$. This, together with (4.4) and (4.5), implies that all the components of the shape operator $A$ are constant along the $\mathcal{D}$-leaves. Moreover, equations (4.6), (4.7) and (4.12) imply that (3.4) with $\alpha+\gamma=\mu$, where $d / d s$ stands for the derivative with respect to $e_{3}$. Note that the existence of such a hypersurface is guaranteed by Example 3.1.

Case (b.2): $a_{11} a_{22}-a_{21} a_{12}=0$ on an open neighborhood $V_{2}$ of a point $p \in V$. In this case, eliminating $\kappa_{1}$ from this condition and (4.17) yields

$$
p_{1}(\gamma, \mu) \omega^{2}+p_{2}(\gamma, \mu) \omega+p_{3}(\gamma, \mu)=0,
$$

where $\omega=\beta^{2}$, and $p_{i}$ are polynomials given by

$$
\begin{aligned}
& p_{1}=16 \gamma-4 \mu, \\
& p_{2}=16 \gamma^{3}-24 \gamma^{2} \mu+8 \gamma \mu^{2}-\mu^{3}-2 \mu, \\
& p_{3}=-\mu\left(2 \gamma^{2}-\gamma \mu-1\right)^{2} .
\end{aligned}
$$

Differentiating (4.27) with respect to $e_{3}$, and using (4.6), (4.16) and (4.20), we obtain

$$
\begin{aligned}
& \kappa_{1}\left(12 \beta^{4}-12 \beta^{4} \mu+24 \beta^{2} \gamma^{3}-56 \beta^{2} \gamma^{2} \mu+37 \beta^{2} \gamma \mu^{2}-2 \beta^{2} \gamma\right. \\
& -5 \beta^{2} \mu^{3}+2 \beta^{2} \mu-4 \gamma^{5}-4 \gamma^{4} \mu+17 \gamma^{3} \mu^{2}+4 \gamma^{3} \\
& \left.-11 \gamma^{2} \mu^{3}+2 \gamma \mu^{4}-6 \gamma \mu^{2}-\gamma+2 \mu^{3}+\mu\right) \\
& +76 \beta^{5} \gamma+16 \beta^{5} \mu+120 \beta^{3} \gamma^{3}-192 \beta^{3} \gamma^{2} \mu+37 \beta^{3} \gamma \mu^{2} \\
& +62 \beta^{3} \gamma-2 \beta^{3} \mu^{3}-20 \beta^{3} \mu+28 \beta \gamma^{5}-152 \beta \gamma^{4} \mu \\
& +169 \beta \gamma^{3} \mu^{2}+36 \beta \gamma^{3}-76 \beta \gamma^{2} \mu^{3}-48 \beta \gamma^{2} \mu+18 \beta \gamma \mu^{4} \\
& +42 \beta \gamma \mu^{2}-\beta \gamma-2 \beta \mu^{5}-10 \beta \mu^{3}-4 \beta \mu=0 .
\end{aligned}
$$

Eliminating $\kappa_{1}$ from (4.28) and (4.17), we get

$$
q_{1}(\gamma, \mu) \omega^{3}+q_{2}(\gamma, \mu) \omega^{2}+q_{3}(\gamma, \mu) \omega+q_{4}(\gamma, \mu)=0,
$$

where $\omega=\beta^{2}$, and $q_{i}$ are polynomials given by

$$
\begin{aligned}
q_{1}= & 88 \gamma+4 \mu, \\
q_{2}= & 168 \gamma^{3}-284 \gamma^{2} \mu+86 \gamma \mu^{2}+48 \gamma-7 \mu^{3}-6 \mu, \\
q_{3}= & 72 \gamma^{5}-292 \gamma^{4} \mu+316 \gamma^{3} \mu^{2}+12 \gamma^{3}-134 \gamma^{2} \mu^{3} \\
& +14 \gamma^{2} \mu+25 \gamma \mu^{4}-3 \gamma \mu^{2}-2 \mu^{5}-3 \mu^{3}-5 \mu, \\
q_{4}= & (\mu-\gamma)\left(2 \gamma^{2}-\gamma \mu-1\right)^{2}\left(2 \gamma^{2}+5 \gamma \mu-2 \mu^{2}-1\right) .
\end{aligned}
$$


The resultant $R_{1}(\gamma, \mu)$ of the left-hand sides of (4.27) and (4.29) with respect to $\omega$ is found to be the following polynomial:

$$
R_{1}(\gamma, \mu)=32(4 \gamma-\mu)\left(2 \gamma^{2}-\gamma \mu-1\right)^{3}\left(1536 \gamma^{8}+\sum_{i=0}^{7} g_{i}(\mu) \gamma^{i}\right)
$$

where $g_{i}$ are polynomials given by

$$
\begin{aligned}
& g_{0}=3 \mu^{8}-8 \mu^{6}+6 \mu^{4}, \\
& g_{1}=-78 \mu^{7}+60 \mu^{5}+52 \mu^{3}, \\
& g_{2}=720 \mu^{6}+204 \mu^{4}+160 \mu^{2}, \\
& g_{3}=-3040 \mu^{5}-1016 \mu^{3}+112 \mu, \\
& g_{4}=6752 \mu^{4}+576 \mu^{2}+32, \\
& g_{5}=-9152 \mu^{3}-608 \mu, \\
& g_{6}=8256 \mu^{2}-192, \\
& g_{7}=-4480 \mu .
\end{aligned}
$$

Case (b.2.i): $4 \gamma-\mu=0$ on an open subset $V_{21} \subset V_{2}$. Differentiating this condition with respect to $e_{3}$, and using (4.6), (4.16) and (4.17), we obtain

$$
6 \gamma^{3}-9 \gamma^{2} \mu+3\left(\mu^{2}+2 \beta^{2}-1\right) \gamma+\left(5 \beta^{2}+3\right) \mu=0 .
$$

Eliminating $\gamma$ from this equation and $4 \gamma-\mu=0$ yields

$$
\mu\left(9 \mu^{2}+208 \beta^{2}+72\right)=0
$$

which shows that $\mu=\gamma=0$ and hence $V_{21}$ is minimal ruled.

Case (b.2.ii): $2 \gamma^{2}-\gamma \mu-1=0$ on an open subset $V_{22} \subset V_{2}$. Differentiating this condition with respect to $e_{3}$, and using (4.6), (4.16) and (4.17), we get

$$
6 \gamma^{4}-11 \gamma^{3} \mu+\left(6 \mu^{2}+6 \beta^{2}-3\right) \gamma^{2}+\left(4 \mu+3 \beta^{2} \mu-\mu^{3}\right) \gamma-\mu^{2}-\beta^{2} \mu^{2}=0 .
$$

Eliminating $\gamma$ from this equation and $2 \gamma^{2}-\gamma \mu-1=0$, we have

$$
\beta^{2}\left(2 \mu^{4}+15 \mu^{2}-9\right)=0,
$$

which implies that $\mu$ is a non-zero constant because of $\beta \neq 0$. However, this contradicts [7, Theorem 1.2]. Therefore, $V_{22}$ is an empty set.

Case (b.2.iii): $f(\gamma, \mu):=1536 \gamma^{8}+\sum_{i=0}^{7} g_{i}(\mu) \gamma^{i}=0$ on an open subset $V_{23} \subset V_{2}$. We differentiate this condition with respect to $e_{3}$, and use (4.6), (4.16) and (4.17). Then, putting $\omega=\beta^{2}$, we obtain

$$
\begin{aligned}
& \omega\left(7808 \gamma^{8}-6464 \gamma^{7} \mu-1856 \gamma^{6} \mu^{2}-1760 \gamma^{6}+19744 \gamma^{5} \mu^{3}-2160 \gamma^{5} \mu\right. \\
& -24576 \gamma^{4} \mu^{4}-2840 \gamma^{4} \mu^{2}+240 \gamma^{4}+16304 \gamma^{3} \mu^{5}+444 \gamma^{3} \mu^{3}+664 \gamma^{3} \mu \\
& +444 \gamma^{3} \mu^{3}+664 \gamma^{3} \mu-5826 \gamma^{2} \mu^{6}-1224 \gamma^{2} \mu^{4}+484 \gamma^{2} \mu^{2}+939 \gamma \mu^{7} \\
& \left.+66 \gamma \mu^{5}+158 \gamma \mu^{3}-51 \mu^{8}+54 \mu^{6}+14 \mu^{4}\right) \\
& +7808 \gamma^{10}-26560 \gamma^{9} \mu+48256 \gamma^{8} \mu^{2}-5664 \gamma^{8}-59296 \gamma^{7} \mu^{3} \\
& +12080 \gamma^{7} \mu+50976 \gamma^{6} \mu^{4}-17256 \gamma^{6} \mu^{2}+1120 \gamma^{6}-31888 \gamma^{5} \mu^{5} \\
& +18356 \gamma^{5} \mu^{3}+360 \gamma^{5} \mu+13998 \gamma^{4} \mu^{6}-11596 \gamma^{4} \mu^{4}-960 \gamma^{4} \mu^{2} \\
& -120 \gamma^{4}-3795 \gamma^{3} \mu^{7}+6138 \gamma^{3} \mu^{5}+434 \gamma^{3} \mu^{3}-208 \gamma^{3} \mu \\
& +528 \gamma^{2} \mu^{8}-2511 \gamma^{2} \mu^{6}-1346 \gamma^{2} \mu^{4}+90 \gamma^{2} \mu^{2}-27 \gamma \mu^{9} \\
& +480 \gamma \mu^{7}+386 \gamma \mu^{5}+200 \gamma \mu^{3}-27 \mu^{8}+6 \mu^{6}+38 \mu^{4}=0 .
\end{aligned}
$$

Computing the resultant of the left hand sides of (4.27) and (4.30) with respect to $\omega$, we obtain

$$
\left(2 \gamma^{2}-\gamma \mu-1\right) \sum_{i=0}^{18} h_{i}(\mu) \gamma^{i}=0
$$


where $h_{i}(\mu)$ are polynomials given by

$$
\begin{aligned}
& h_{0}=1377 \mu^{19}-4527 \mu^{17}+1284 \mu^{15}+4728 \mu^{13} \\
& +1468 \mu^{11}-4908 \mu^{9}, \\
& h_{1}=-66060 \mu^{18}+143388 \mu^{16}+45504 \mu^{14}-74376 \mu^{12} \\
& -90176 \mu^{10}-24512 \mu^{8} \\
& h_{2}=1397709 \mu^{17}-1823607 \mu^{15}-1459380 \mu^{13}-146496 \mu^{11} \\
& +289596 \mu^{9}+119828 \mu^{7} \\
& h_{3}=-17308746 \mu^{16}+11826810 \mu^{14}+14566216 \mu^{12}+6035952 \mu^{10} \\
& +2043336 \mu^{8}+763816 \mu^{6}, \\
& h_{4}=140724708 \mu^{15}-40031364 \mu^{13}-73716152 \mu^{11}-29812064 \mu^{9} \\
& -6305728 \mu^{7}+511024 \mu^{5}, \\
& h_{5}=-801068376 \mu^{14}+56606496 \mu^{12}+232622128 \mu^{10}+61803936 \mu^{8} \\
& -3732032 \mu^{6}-1748096 \mu^{4}, \\
& h_{6}=3336681024 \mu^{13}+31432448 \mu^{11}-555418672 \mu^{9}-78779056 \mu^{7} \\
& +7995776 \mu^{5}-2476096 \mu^{3}, \\
& h_{7}=-10529445888 \mu^{12}-118321664 \mu^{10}+1089457312 \mu^{8}+68763808 \mu^{6} \\
& +4847488 \mu^{4}-430976 \mu^{2} \\
& h_{8}=25909096832 \mu^{11}-541759232 \mu^{9}-1652978624 \mu^{7}+6159040 \mu^{5} \\
& +14641152 \mu^{3}+625920 \mu, \\
& h_{9}=-50856105728 \mu^{10}+3160585216 \mu^{8}+1852903808 \mu^{6}-128791552 \mu^{4} \\
& +12595200 \mu^{2}+230400 \\
& h_{10}=80930532864 \mu^{9}-8199388160 \mu^{7}-1406354176 \mu^{5}+71124736 \mu^{3} \\
& -3322880 \mu \text {, } \\
& h_{11}=-105451162624 \mu^{8}+14029552640 \mu^{6}+551636480 \mu^{4}-60403200 \mu^{2} \\
& -3379200 \text {, } \\
& h_{12}=112905166848 \mu^{7}-17520074752 \mu^{5}+317690880 \mu^{3}-40262656 \mu \text {, } \\
& h_{13}=-98991538176 \mu^{6}+16388972544 \mu^{4}-537110528 \mu^{2}+27381760 \text {, } \\
& h_{14}=70233264128 \mu^{5}-11463987200 \mu^{3}+441262080 \mu \text {, } \\
& h_{15}=-39343300608 \mu^{4}+5679833088 \mu^{2}-109936640 \text {, } \\
& h_{16}=16633511936 \mu^{3}-1843052544 \mu \text {, } \\
& h_{17}=-4831674368 \mu^{2}+243859456 \text {, } \\
& h_{18}=767557632 \mu \text {. }
\end{aligned}
$$

Since Case (b.2.ii) does not occur, we have $\sum_{i=0}^{18} h_{i}(\mu) \gamma^{i}=0$. The resultant $R_{2}(\mu)$ of $f(\gamma, \mu)$ and $\sum_{i=0}^{18} h_{i}(\mu) \gamma^{i}$ with respect to $\gamma$ is given by

$$
R_{2}(\mu)=\mu^{36} k(\mu),
$$

where $k(\mu)$ is a polynomial in $\mu$ with constant coefficients of degree 116. Since the explicit form of $k(\mu)$ is not important for the argument, we do not list it. Thus, we deduce that $\mu$ is constant, that is, the mean curvature is constant. According to [7, Theorem 1.2], we conclude that $V_{23}$ is minimal ruled.

Consequently, $M$ is locally obtained by the construction described in Example 3.1. The proof is finished.

\section{References}

[1] Cecil, T. E., Ryan, P. J.: Geometry of Hypersurfaces. Springer Monographs in Mathematics. Springer, New York (2015). 
[2] Chen, B. Y.: Some pinching and classification theorems for minimal submanifolds. Arch. Math. (Basel) 60, 568-578 (1993).

[3] Chen, B. Y.: A general inequality for submanifolds in complex space forms and its applications. Arch. Math. (Basel) 67, 519-528 (1996).

[4] Chen, B. Y.: Pseudo Riemannian Geometry, $\delta$-invariants and Applications. World Scientific, Hackensack, New Jersey (2011).

[5] Ivey, T. A., Ryan, P. J.: Hypersurfaces in $\mathbb{C} P^{2}$ and $\mathbb{C} H^{2}$ with two distinct principal curvatures. Glasgow Math. J. 58, 137-152 (2016).

[6] Kimura, M.: Sectional curvatures of holomorphic planes on a real hypersurface in $P^{n}(\mathbb{C})$. Math. Ann. 276, 487-497 (1987).

[7] Sasahara, T.: Real hypersurfaces in the complex projective plane attaining equality in a basic inequality. Houston J. Math. 43, 89-94 (2017).

\section{Affiliations}

TORU SASAHARA

AdDress: Center for Liberal Arts and Sciences, Hachinohe Institute of Technology, Hachinohe, Aomori 0318501, Japan

E-MAIL: sasahara@hi-tech.ac.jp

ORCID ID: 0000-0003-2853-0268 